\title{
Aphasie : Contraintes vs Gouvernement Le cas des clusters
}

\author{
Prince, Typhanie \& Tifrit, Ali \\ Laboratoire de Linguistique de Nantes, Lling EA 3827. \\ typhanie.prince@univ-nantes.fr \\ ali.tifrit@univ-nantes.fr
}

\section{Introduction}

Ce travail propose une analyse des processus phonologiques en jeu dans l'aphasie, en particulier, à travers l'étude des groupes consonantiques dans les structures syllabiques dont ils dépendent. Dans cette optique, nous développons une approche unifiée des domaines phonologiques et aphasiologiques (Wilshire C. \& Nespoulous J-L., 2003) qui prend en compte :

- la dimension segmentale, à travers la notion de complexité (Harris, 1994), (Scheer, 1998) (Angoujard, 1997) ;

- la dimension syllabique, par le biais du gouvernement et des contraintes qu'il impose sur les représentations phonologiques (Scheer, 2010).

Les approches récentes de Den Ouden (2002), Valdois (2006) ou encore Prince (2011), montrent que les deux niveaux, segmental et syllabique, entrent en jeu dans l'étude des aphasies. Nous postulons que ce n'est qu'en considérant leur interrelation que l'on peut produire une explication des désordres phonologiques observés en pathologie et montrer que des régularités émergent d'une situation considérée comme a priori chaotique.

Le cas des clusters consonantiques apparaît comme un point d'ancrage dans cette problématique. Paradoxalement, cet objet n'a pas, ou peu, été exploité dans les travaux aphasiologiques alors qu'il apparaît central dans les approches phonologiques par les problèmes représentationnels qu'il pose (Laks, 2005, Brandão \& Wauquier, 2007) : constituance, relations ou absence de relations entre les consonnes dans une même structure, hétérosyllabicité ou ambisyllabicité. Dans cette phénoménologie, certains clusters revêtent un statut particulier (Kaye, 1996) et font surfacer, dans notre analyse des cas pathologiques, cette relation entre éléments segmentaux et structure syllabique : il s'agit des groupes /s+occlusive/ et /s+occlusive/.

Nous présenterons en section 2, comme outils d'analyse des aphasies, les cadres phonologiques exploitant la notion de Contrainte : la Théorie des Contraintes et des Stratégies de Réparation (désormais TCSR) et la Théorie de l'Optimalité (dorénavant OT). Par la suite, nous introduirons les notions de Gouvernement et de Licenciement qui constituent les concepts centraux de la Théorie du Gouvernement (CVCV). En section 3, nous observerons quels types de traitements proposent la TCSR et OT. Nous verrons ce que ces modèles apportent à l'aphasiologie et soulignerons les apories qu'ils rencontrent. Nous expliciterons dans la section 4 la méthode utilisée pour recueillir les données et l'analyse que nous défendons dans le cadre de la Phonologie du Gouvernement (Scheer, 2004, 2010), cadre dans lequel nous nous inscrivons. Nous y montrerons comment le modèle $C V C V$ répond à la question des groupes consonantiques et nous discuterons des conséquences des analyses sur les clusters. Nous montrerons comment les résultats obtenus ouvrent sur de nouvelles perspectives concernant la complexité. Enfin, nous discuterons en 5 les apports de cette étude et les directions futures. 


\section{Aphasiologie : des Contraintes au Gouvernement}

Deux cadres phonologiques, exploités pour l'étude de l'aphasie, manipulent la notion de Contrainte : TCSR et OT. Malgré quelques divergences théoriques, ces approches semblent aboutir à des conclusions similaires concernant les stratégies développées par les patients aphasiques, comme nous le verrons plus loin. Un autre cadre phonologique manipule quant à lui la notion de Gouvernement : CVCV. Ce dernier ouvre de nouvelles perspectives dans l'étude et l'explication des processus aphasiques. Notre objectif consiste à confronter les approches par Contraintes et Gouvernement et à démontrer en quoi la Phonologie du Gouvernement, spécifiquement le modèle CVCV, constitue une réponse inédite aux phénomènes et stratégies développés dans l'aphasie. Nous commençons d'abord par expliciter chacun de ces cadres théoriques.

\subsection{TCSR : Principes et Paramètres}

La théorie générative, dans sa déclinaison "Principes et Paramètres", postule que la Grammaire Universelle (GU), constituée d'universaux, reflète les caractéristiques communes aux langues du monde. Ces principes, en nombre fini, définissent la totalité des langues humaines. A ces principes universels s'ajoutent des paramètres qui peuvent être fixés négativement ou positivement et qui définissent des propriétés spécifiques à chaque langue. Ces paramètres sont fixés lors de l'acquisition du langage pour tout locuteur membre d'une communauté linguistique donnée : dans le cadre de la Théorie des Contraintes et des Stratégies de Réparation, les paramètres constituent des contraintes. Par exemple, si universellement toutes les langues possèdent des syllabes (Principe), il n'est pas vrai qu'elles possèdent toutes des syllabes CVC, c'est-à-dire, avec une Coda (Paramètre).

Les travaux de N. Moreau (1993), S. Valdois $(1987,2006)$ et ceux de R. Béland \& C. Paradis (1997) exploitent cette notion de Contrainte, tant au niveau segmental qu'aux niveaux des structures syllabique et prosodique, dans une hiérarchie universelle qui n'est, de fait, pas spécifique aux sujets aphasiques. Dans la grammaire interne du sujet non-aphasique, cette hiérarchie correspond à l'appropriation de plusieurs strates acquises graduellement et de complexité croissante : du segment au syntagme phonologique.

Concernant les aphasiques, ces auteurs postulent que les transformations opérées (particulièrement les paraphasies phonémiques) ne correspondent pas à des erreurs à proprement parler puisqu'elles seraient produites en respectant des contraintes phonologiques attestées dans d'autres langues du monde. Si cette prédiction se révèle correcte, les auteurs suggèrent que les aphasiques produiront plus d'erreurs au sein des contextes marqués (qui sont plus complexes), dans des structures syllabiques de type CVC ou encore $\mathrm{CCVC}$, ne correspondant donc pas à des structures de type $\mathrm{CV}$, non marquées (non complexes).

Dans cette optique, trois principes ${ }^{1}$ majeurs sont à retenir :

1- La stratégie de réparation est une opération phonologique universelle consistant à insérer ou élider du matériel linguistique afin de satisfaire une contrainte violée. La présence de ce matériel phonologique se justifie afin de combler un manque susceptible d'engendrer une violation de contrainte.

2- Le principe de préservation consiste à limiter les pertes d'informations segmentales afin de préserver maximalement les informations dans la limite du conflit. Ce principe est conjoint au principe du seuil de tolérance qui a pour effet de dissiper et d'atténuer les mécanismes de préservation. Ce principe du seuil de tolérance précise que :

(i) toutes les langues établissent une limite à la préservation segmentale et que,

(ii) cette limite est de $\mathrm{X}$ étapes dans un domaine de contrainte donné, $\mathrm{X}$ ne doit en aucun cas dépasser la capacité d'un adulte à reconnaître un patron canonique de nombre. minimale

3- Le principe de minimalité établit qu'une stratégie de réparation doit s'appliquer de façon 
(i) Une stratégie de réparation doit s'appliquer au niveau le plus bas auquel fait référence la contrainte violée ${ }^{2}$.

(ii) Une réparation doit impliquer le moins de stratégies possibles.

Les travaux en TCSR émettent l'hypothèse que les transformations sont toujours gouvernées par le système phonologique : insertion, omission et substitution sont des stratégies de réparation engendrées par le sujet afin de "simplifier" un contexte linguistique.

\subsection{Théorie de l'Optimalité (OT)}

Dans les travaux en phonologie post-générative, OT conçoit le système linguistique comme un ensemble de contraintes universelles hiérarchisées. Chaque langue possède sa propre hiérarchie de contraintes. Celles-ci doivent être ordonnées de manière à rendre compte des phénomènes propres au système phonologique considéré. Il s'agit de comprendre quels sont les processus qui permettent de sélectionner une représentation de surface bien formée. De comprendre pourquoi celle-ci est sélectionnée au détriment d'une autre. OT est donc une théorie de l'interaction des contraintes basée sur l'évaluation des formes de surface.

On distingue trois composantes de base pour la théorie : Un générateur (GEN) génère un vaste ensemble de candidats possibles en output qui seront sélectionnés à partir de leur proximité à une forme sousjacente donnée. Chacun d'entre eux est potentiellement un candidat optimal, c'est-à-dire un candidat susceptible d'être généré en surface. Ces formes sont ensuite soumises à un évaluateur (EVAL) qui prend en charge l'évaluation des différents candidats. Il s'agit d'éliminer les formes indésirables au filtre des contraintes et de faire surfacer la forme optimale souhaitée. Cette évaluation est réalisée, sur la base du nombre de transgressions / violations de ces multiples contraintes, qui permet d'exclure les formes indésirables générées par GEN. Un dernier composant, $\mathrm{CON}$, fournit les critères de contraintes et classe les différentes contraintes nécessaires à l'évaluation.

Les contraintes se classent en deux grandes familles :

- Contraintes de Marque : renvoient à des tendances observées dans les systèmes phonologiques,

- Contraintes de Fidélité : établissent la distance observée entre l'input et l'output.

Les contraintes de Marque entrent en compétition avec les contraintes de Fidélité dans l'établissement de la hiérarchie. Les candidats qui ont été générés sont ensuite comparés et évalués.

Ces contraintes sont souvent contradictoires et de fait en conflit permanent, elles sont permutables et violables, contrairement à la TSCR où les contraintes violées donnent naissance à des stratégies de réparation. La réorganisation des contraintes dans la hiérarchie modifie le résultat en output et, par conséquent, le candidat optimal. Les contraintes s'expriment toujours selon un ordre précis : à droite se situent les contraintes de rang inférieur, celles-ci ont un poids moindre que les contraintes placées à gauche. Le candidat qui ne viole pas de contrainte ou qui viole des contraintes placées le plus à droite figure comme candidat optimal.

Les travaux de Den Ouden (2002) dans ce cadre portent sur l'étude des paraphasies et des choix opérés dans la sélection des différentes formes phonologiques. Selon l'auteur, OT est capable de fournir une représentation intuitive de la genèse des troubles phonologiques et peut rendre compte de la variation d'output dans l'aphasie. 


\subsection{Théorie du Gouvernement (CVCV)}

La Théorie du Gouvernement, depuis Kaye \& Lowenstamm (1981), KLV (1985), Kaye (1996) et jusqu'aux propositions récentes de Lowenstamm (1996) et Scheer (2004, 2010), postule, à l'instar de la TCSR, que la structure syllabique d'une langue donnée est le résultat d'une combinaison de principes universels et de paramètres propres à une langue.

Le cadre $\mathrm{CVCV}$, en continuité des travaux de $\mathrm{KLV}$, propose que toute représentation syllabique répond à la stricte consécution d'un schème $\mathrm{CV}$. Ce schème correspond à la syllabe minimale et universelle (c'est-àdire la moins marquée). La phonologie est régie par des relations de gouvernement (propre et infrasegmental) et de licenciement. L'idée fondamentale du cadre CVCV est la latéralité où il n'y a qu'une seule strate : les éléments ont des relations latérales et communiquent les uns avec les autres par l'intermédiaire de conditions de gouvernement et de licenciement. La syllabe n'est donc pas régie, contrairement à OT, par une hiérarchie de contraintes. Chaque consonne est nécessairement suivie d'une voyelle ce qui entraîne la présence de noyaux vides dans la représentation sous-jacente. Gouvernement et Licenciement sont des conditions qui limitent cette prolifération d'éléments non réalisés segmentalement. Autrement dit, il n'y a pas forcément d'identité entre représentation sous-jacente et représentation de surface.

A ce titre, le Gouvernement Propre (GP) est une condition sur l'expression ou l'inhibition des voyelles : une voyelle $(\mathrm{V})$ peut ne pas être réalisée segmentalement (Ø) si elle est proprement gouvernée par le vocoïde qui suit. Le Gouvernement Infra-segmental (GI), quant à lui, établit une relation interconsonantique. Son rôle est de constituer un domaine de gouvernement qui rend " invisible " la voyelle ( $\mathrm{V}_{2}$ dans le schéma (1) suivant) entre deux consonnes sujettes à cette relation et autorise la voyelle suivante $\left(V_{3}\right)$ à gouverner la voyelle qui précède ce domaine ( $V_{1}$ dans le schéma (1) suivant).

(1) Gouvernement propre et infra-segmental

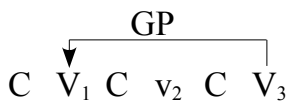

$$
\begin{aligned}
& \mathrm{C} \varnothing(\mathrm{T} \leftarrow \mathrm{R}) \mathrm{V} \\
& \text { GI Lic }
\end{aligned}
$$

Où $\mathrm{T}$ renvoie à tous types d'obstruantes et où $\mathrm{R}$ représente les sonantes. Le gouvernement infra-segmental s'applique toujours de droite à gauche. La sonante gouverne l'obstruante du fait de sa complexité.

Enfin, le Licenciement (Lic) est une condition d'ordre syntagmatique permettant l'interaction entre consonne. Par exemple, $\mathrm{R}$ dans le cas précédent est autorisé à gouverner T parce qu'il est licencié par la voyelle $\mathrm{V}_{3}$ qui suit.

(2) Licenciement et gouvernement infra-segmental

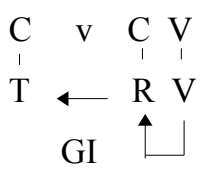

Lic

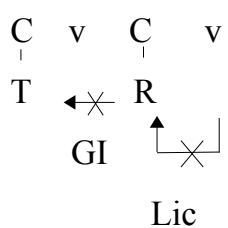

Lic

Le gouvernement infra-segmental peut s'établir seulement si la consonne qui gouverne les constituants vides est licenciée par son propre noyau et seul un noyau réalisé phonétiquement est un licencieur possible (exception faite de la paramétrisation du noyau vide final).

Les travaux en Phonologie du Gouvernement de Valdois (1987) et Valdois \& Nespoulous (1994) présentent une analyse des aphasies basée sur le modèle du Charme et du Gouvernement de KLV. Ils montrent entre autres que dans les stratégies adoptées, ce sont les constituants en position branchante (gouvernée) qui font le plus souvent l'objet d'omission. Ici aussi, tout converge donc vers une 
simplification des structures syllabiques. Or, un traitement des désordres phonologiques aphasiques au filtre d'un modèle phonologique tel que $\mathrm{CVCV}$, outre le fait qu'il n'a jamais été exploité en aphasiologie, apparaît en mesure d'expliquer des phénomènes engendrés par l'aphasie qui, jusqu'à présent, ne bénéficiaient pas d'un traitement satisfaisant notamment au regard de la question de la complexité. Ce type d'analyse permet d'observer la nature des transformations mais également d'expliquer le fonctionnement de chacun des processus opérés parce qu'il définit des opérations actives à la fois aux niveaux segmental et syllabique. Nous suggérons que CVCV va, de fait, rendre compte des mécanismes linguistiques à l'oeuvre dans l'aphasie.

\section{Traitement par Contraintes : TCSR et OT}

L'approche par Contraintes développées par la TCSR et la Théorie de L'Optimalité prend pour acquis que le désordre aphasique engendre un bouleversement de la hiérarchie des contraintes. Ces études sont basées particulièrement sur la réduction des clusters consonantiques où les données sont issues de populations aphasiques fluentes et non-fluentes. Si les deux cadres semblent converger vers la même solution, celle où les paraphasies résultent effectivement de la violation de contraintes sanctionnant l'usage de constituants complexes, ils ne sauraient être considérés comme équivalents. En effet, le premier pose des conditions sur une structure hiérarchisée (une hypothèse de profondeur structurelle, qui n'est pas réductible à la surface) alors que le second ne fait état que de rapports de contiguïté séquentielle (une hypothèse linéaire : où la succession des éléments en surface constitue l'unique moteur de l'explication).

\subsection{Contraintes et Stratégies}

Les travaux de Moreau (1993), Valdois (1986) et Béland \& Paradis (1997) dans le cadre de la TCSR ont pour objectif de déterminer les positions syllabiques les plus sujettes aux paraphasies phonémiques. Les auteurs considèrent, après analyse des données, que les désordres phonologiques dans l'aphasie sont le résultat de mauvaises fixations de paramètres. Par exemple, le paramètre épenthèse vocalique (insertion) dans un groupe /occlusive + liquide/ est fixé négativement en français et positivement en peul dans le cadre des emprunts puisque cette langue n'admet pas ce type d'attaques complexes :

$$
\text { (3) fr. bleu [blø] et pas *[bəlø] emprunt en peul [bəlø] et pas *[blø] }
$$

Un aphasique locuteur du français aura tendance à fixer positivement ce paramètre à l'instar des locuteurs du peul et produira [bəlø] pour fr. bleu : autrement dit le groupe $/ \mathrm{CC} /$ sera transformé en $/ \mathrm{CəC} /$. Les transformations opérées dans ces clusters par le sujet aphasique constituent une stratégie de réparation qui fait suite à la violation d'une contrainte structurale d'ordre syllabique.

C'est aussi le cas dans ce qui apparaît, en (4), comme une réduction des attaques branchantes :

(4) patrie : /patsi/ > [pati $]^{3}$

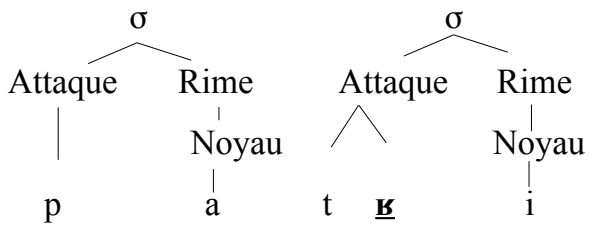

parti : /pasti/ > [pati]

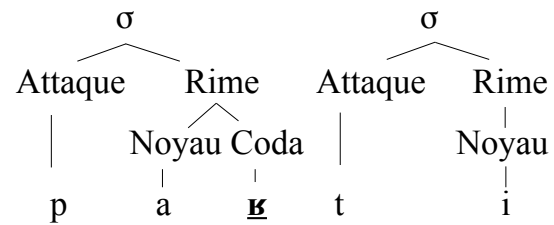

Ce dysfonctionnement phonologique, selon N. Moreau (1993), correspond à une simplification des structures complexes. Cette simplification est due à une fixation positive ou négative, de contraintes chez l'aphasique. Les transformations constatées ne correspondent donc pas à des erreurs à proprement parler puisqu'elles seraient produites en respectant des contraintes phonologiques attestées par ailleurs dans d'autres langues du monde. En d'autres termes, les aphasiques ne seraient plus capable d'édifier des structures complexes car cette fixation de contraintes conduit à des possibilités restreintes aux niveaux segmental et/ou syllabique. 


\subsection{Optimalité}

D-B. Den Ouden (2002) propose plus récemment une analyse de la réduction des clusters dans le cas des monosyllabes. De la même manière que Moreau, Béland \& Paradis, il observe comment les paraphasies agissent au niveau syllabique et quelles sont les positions les plus atteintes. Il postule aussi que dans l'aphasie, tout comme dans le langage non-pathologique, différentes contraintes peuvent entrer en conflit, ce sont ces contraintes universelles qui régissent le système phonologique. L'auteur propose d'en observer le fonctionnement et de comparer le devenir des clusters chez les aphasiques fluents et non-fluents.

D'après Den Ouden, un item print/print/ sera produit majoritairement [pit] par des aphasiques non-fluents alors que des aphasiques fluents peuvent produire indifféremment les deux formes [pin] et [pit]. Afin de prédire ces variations dans les réalisations, Den Ouden (2002) emploie les contraintes suivantes qui se répartissent en deux catégories : des contraintes de Marque, qui renvoient à des tendances observées dans les systèmes phonologiques, et, une contrainte de Fidélité, qui établit la distance observée entre l'input, ou la sortie attendue, et l'output, la sortie effective :

Contraintes de Marque :

*C[+son] rejeter les sonantes (les consonnes sont idéalement [-sonant]).

HOns (Onset Harmony) l'attaque doit présenter un accroissement abrupt de sonorité / les attaques ne doivent pas être sonantes.

HCod (Coda Harmony) la coda ne doit pas présenter de diminution abrupte de la sonorité / elle doit être préférentiellement une sonante.

Contraintes de Fidélité :

Parse

préservation maximale du matériel en input.

Ainsi, les contraintes dessinent une syllabe idéalisée dont la structure pourrait se résumer ainsi : éviter les constituants complexes et sélectionner des catégories segmentales spécifiques pour l'attaque et la coda.

(5) Syllabe idéale

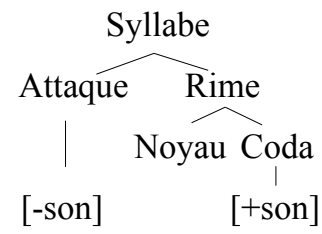

Den Ouden propose deux hiérarchies de contraintes correspondant à la grammaire des sujets aphasiques. Celles-ci rendent compte de la variation d'output observée :

(a) aphasie fluente et non-fluente ${ }^{4}$

(b) aphasie fluente

$$
\begin{aligned}
& * \mathrm{C}[+ \text { son }]>\text { HCod }>>\text { HOns }>>\text { Parse } \\
& \mathrm{HCod}>>* \mathrm{C}[+ \text { son }]>\text { HOns }>>\text { Parse }
\end{aligned}
$$

Les hiérarchies proposées dans les tableaux (a) et (b) suivants, où l'ordre des contraintes $* \mathrm{C}[+$ son $]$ et HCod est décisif, permettent de sélectionner le candidat optimal pour chaque type d'aphasie : 
(a) /print/ $\rightarrow$ [pit] / aph. non-fluente et fluente

\begin{tabular}{|c|c|c|c|c|}
\hline /print/ & $*$ C [+son] & HCod & HOns & Parse \\
\hline print & $* * !$ & $*$ & $*$ & \\
\hline pint & $* !$ & $*$ & & $*$ \\
\hline pit & & $*$ & & $* *$ \\
\hline pin & $* !$ & & & $* *$ \\
\hline rit & $* !$ & $*$ & $*$ & $* *$ \\
\hline rin & $* * !$ & & $*$ & $* *$ \\
\hline rint & $* * !$ & $*$ & $*$ & $*$ \\
\hline prit & $* !$ & $*$ & $*$ & $*$ \\
\hline prin & $* * !$ & & $*$ & $*$ \\
\hline
\end{tabular}

(b) /print/ $\rightarrow$ [pin] / aph. fluente

\begin{tabular}{|c|c|c|c|c|}
\hline /print/ & HCod & $*$ C [+son] & HOns & Parse \\
\hline print & $* !$ & $* *$ & $*$ & \\
\hline pint & $* !$ & $*$ & & $*$ \\
\hline pit & $* !$ & & & $* *$ \\
\hline pin & & $*$ & & $* *$ \\
\hline rit & $* !$ & $*$ & $*$ & $* *$ \\
\hline rin & & $* * !$ & $*$ & $* *$ \\
\hline rint & $* !$ & $* *$ & $*$ & $*$ \\
\hline prit & $* !$ & $*$ & $*$ & $*$ \\
\hline prin & & $* * !$ & $*$ & $*$ \\
\hline
\end{tabular}

Les deux contraintes de Marque ${ }^{*} \mathrm{C}[+\mathrm{s}$ son] et Hcod ne font, contrairement à ce que l'on pourrait penser, nullement référence à des conditions de structure : elles renvoient toutes deux au matériel segmental manipulé et à l'ordre d'apparition de ces segments, reprenant implicitement l'Absolute Slicing Hypothesis (Goldsmith,1990), c'est-à-dire une hypothèse où la syllabe n'existe tout simplement pas.

En appliquant les hiérarchies de contraintes proposées par Den Ouden (2002) aux données de Prince (2011), on s'aperçoit qu'elles aboutissent à des résultats paradoxaux. Les tableaux (c) et (d) suivants traitent l'item escargot /eskabgo/ (Prince, 2011) qui comporte un certain nombre de constituants problématiques : une attaque nulle, un groupe /s+occlusive/ et un cas / $\mathrm{b}+$ occlusive/.

(c) escargot / eskargo/ aph. non-fluente / fluente

\begin{tabular}{|c|c|c|c|c|}
\hline /eskasgo/ & $* \mathrm{C}[+\operatorname{son}]$ & HCod & HOns & Parse \\
\hline عкавgo & *! & & & $*$ \\
\hline عsaьgo & *! & & & * \\
\hline Eskago & & $* !$ & & $*$ \\
\hline عskаьо & *! & * & * & * \\
\hline عзаво & $* !$ & & * & ** \\
\hline Esago & & & & $* *$ \\
\hline єкаьо & *! & & $*$ & $* *$ \\
\hline ekago & & & & $* *$ \\
\hline кавgо & $* !$ & & & $* *$ \\
\hline kago & & & & $* * * !$ \\
\hline sавgо & *! & & & $* *$ \\
\hline sago & & & & $* * * !$ \\
\hline
\end{tabular}

(d) escargot /eskargo/ aph. fluente

\begin{tabular}{|c|c|c|c|c|}
\hline /عskargo/ & HCod & ${ }^{*} \mathrm{C}[+\operatorname{son}]$ & HOns & Parse \\
\hline عкакgо & & $* !$ & & * \\
\hline عsabgo & & $* !$ & & $*$ \\
\hline Eskago & $* !$ & & & $*$ \\
\hline عskаьо & $* !$ & $*$ & $*$ & $*$ \\
\hline عзаьо & & $* !$ & * & $* *$ \\
\hline Esago & & & & $* *$ \\
\hline єкаьо & & $* !$ & * & ** \\
\hline$\approx$ ckago & & & & $* *$ \\
\hline кавgо & & $* !$ & & $* *$ \\
\hline kago & & & & $* * * !$ \\
\hline sавgo & & $* !$ & & $* *$ \\
\hline sago & & & & $* * * !$ \\
\hline
\end{tabular}


Notons que, sans générer tous les candidats "possibles", l'on rencontre un grand nombre de candidats optimaux qui ne sont départagés que par la contrainte Parse, la plus basse dans la hiérarchie, contrainte qui ne fait que constater que les formes produites ne correspondent pas à la forme attendue. Demeurent, dans tous les cas, deux candidats optimaux : [esago] et [ekago] où seule une contrainte sur le premier segment consonantique qui, par exemple, rejetterait la présence d'une fricative en position d'attaque $(*$ Ons $[+$ fric $])$ pourrait éventuellement aboutir à un résultat non ambigu.

\subsection{Apories}

Ainsi, pour OT, quel que soit l'ordre des contraintes proposées, les hiérarchies de contraintes sont impuissantes à différencier les deux types d'aphasies : les candidats optimaux sont identiques dans les deux tableaux, ce qui suggère que les contraintes utilisées ne sont pas suffisamment restrictives.

Un point important est que les productions des patients ne répondent pas aux résultats générés ici. La majorité des patients produit la forme [Eskago] qui est éliminée par la contrainte HCod et qui de fait infirme l'analyse présentée dans le cadre optimaliste : la forme en question maintient /s/ en coda, bien qu'elle n'appartienne pas à la catégorie sonante, et rejette / $/$ / pourtant optimal dans cette position. Certains

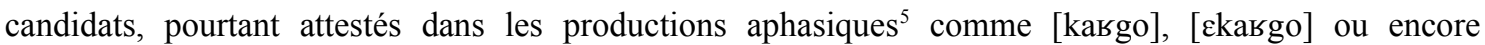
[عskago], ne sont pas sélectionnés au profit d'autres formes comme [sago], [عsago] ou encore [kago] non attestées. La hiérarchie que propose Den Ouden (2002) ne semble s'appliquer qu'à un sous-ensemble de données aphasiques et n'explique pas la majorité des données rencontrées dans l'aphasie, fluente ou nonfluente.

Même si la Théorie de l'Optimalité permet de décrire parfois élégamment les données observées, elle n'explique ni la nature des transformations, ni pourquoi certains candidats optimaux sont préférentiellement adoptés par certains aphasiques et pas par d'autres. En outre, elle ne définit pas les raisons qui font que telle position syllabique est sujette, plutôt que telle autre, à la transformation, et de la même manière, pourquoi tel segment est élidé plutôt qu'un autre.

Cette difficulté peut s'expliquer par l'absence de traitement du niveau syllabique au seul profit de contraintes segmentales agissant de manière totalement autonome. Or, nous plaidons pour l'interdépendance de ces deux niveaux de représentation : seule cette relation permet de produire une explication cohérente des phénomènes. En résumé, les contraintes proposées par Den Ouden dans le cadre de la Théorie de l'Optimalité ne semblent pas en mesure de couvrir les données paraphasiques, à moins de recourir à des contraintes $a d$ hoc ou à des mécanismes qui contredisent les faits et ne permettent pas de comprendre le comportement régulier des aphasiques dans leurs productions. C'est pour ces raisons que nous nous tournons à présent vers la Phonologie du Gouvernement.

\section{Traitement par Gouvernement : CVCV}

Nous savons désormais que le sujet met en place différents mécanismes et stratégies lors de son aphasie. Toutefois, comme nous l'avons montré, la TCSR, si elle explique la nature des processus, ne décrit pas explicitement les mécanismes mis en oeuvre. OT, quant à elle, ne rend pas toujours compte du type de processus opéré et ne fournit pas d'explication quant aux stratégies préférentiellement adoptés. Nous nous tournons, après avoir décrit la méthode suivie, vers l'analyse dans le cadre de la Phonologie du Gouvernement qui révèle cette interdépendance des deux niveaux dans les paraphasies.

\subsection{Méthode}

Notre étude est basée sur un échantillon de 15 patients aphasiques (6 Broca, 7 Wernicke, 2 conduction) de l'unité Neuro-Vasculaire du CHU de Nantes : ces patients souffrent d'un trouble du langage consécutif à 
une lésion cérébrale de l'hémisphère gauche. Les données ont été récoltées dans un maximum de $72 \mathrm{~h}$ après un traumatisme.

Un protocole est élaboré spécifiquement pour faire émerger les paraphasies phonémiques qui touchent les groupes consonantiques à travers une tâche de dénomination et de répétition. Nous avons testé 25 items contenant des groupes de consonnes en position initiale, médiane et finale ( $c f$. tableau (e) suivant).

(e) répartition des clusters et position

\begin{tabular}{|c|c|c|c|}
\hline & Position initiale & Position médiane & Position finale \\
\hline & \#CCv.c & c.CCv ; v.CCv. ; vC.Cv & vCC\# \\
\hline occ + liq & 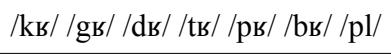 & $/ \mathrm{t} \mathrm{s} / / \mathrm{kl} / / \mathrm{bu} / / \mathrm{b} \boldsymbol{} / / \mathrm{bl} /$ & \\
\hline fric +liq & $/ \mathrm{fu} / / \mathrm{fl} /$ & & \\
\hline $\operatorname{liq}+$ occ & & /в.g//в.b/ /в.t//в.d//в.p/ & /st//sk//sb/ \\
\hline fric +oce & $/ \mathrm{st} /$ & /s.k//s.t//s.k//s.p/ & \\
\hline occ + fric & & /k.s/ & \\
\hline
\end{tabular}

: pas d'image présentée pour ce cas.

: non-attesté.

Ce test constitue notre base d'expérimentation. Nous nous sommes intéressés à l'ensemble des troubles phonologiques pour le cas de la paraphasie : omission, insertion, substitution et permutation, à la fois dans les groupes consonantiques et dans le cas des segments isolés. Nous avons également analysé les items qui présentaient plusieurs transformations phonologiques.

Nous nous intéresserons ici aux cas / $\mathbf{b}+$ occlusive/ et $/ \mathbf{s}+$ occlusive/ parce qu'ils présentent un comportement particulier, à partir des items suivants classés selon le lieu d'articulation de l'occlusive :

(f) clusters et lieux d'articulation

\begin{tabular}{|c|c|c|}
\hline Lieu & /в+occlusive/ & $/ \mathbf{s}+$ occlusive/ \\
\hline [dorsal] & parc /paьk/, escargot /عs.как.go/ & casquette /kas.ket/, escargot /es.kaь.go/ \\
\hline [labial] & arbre /ак.bьә/, serpent /sєь.ра̃/ & aspirateur /as.pi.ьа.tœь/ \\
\hline [coronal] & tortue /tов.ty/, ordinateur /ок.di.na.tœь/ & moustique/mus.tik/ \\
\hline
\end{tabular}

\section{2 Étude de cas}

Nous commencerons par étudier les processus opérés par les patients dans le cas / $\mathrm{b}+$ occlusive/ puis nous nous tournerons vers l'analyse des cas /s + occlusive/. Nous avons uniquement retenu les processus de transformations les plus fréquents, autrement dit, les processus adoptés préférentiellement par les patients.

\section{/в + occlusive/}

Le GP peut rendre compte simplement des cas / $\mathbf{b}+$ occlusive/. Dans les exemples $(6,7$ et 8$)$ suivants, le type / $\mathbf{b}+$ occlusive/ est souvent réalisé sans la liquide dans les productions aphasiques.

Ainsi : (6) [вt] : tortue est réalisé [koty], [tugœ] ${ }^{6}$, mais aussi [tовg], [tuвg], [tвоty], [tвоtœ], [тотуь].

(7) [ьр] : serpent est réalisé [ерã], [tœpã], [scpã] et [sєьәра̃].

(8) [вg] : escargot peut être réalisé [eskago], mais aussi [eskabõ], [kargo], [عkaьgo].

Si dans nombre de ces cas la liquide disparaît, c'est parce qu'elle est située en position faible. Cette position de coda est, pour simplifier, propice à la disparition. La position inter-consonantique ne peut 
s'exprimer parce qu'elle est proprement gouvernée par la voyelle suivante, de fait elle ne peut licencier la consonne $\mathrm{C}_{1}$ qui la précède : elle est en position coda.

(9) positions de lénition et fortition

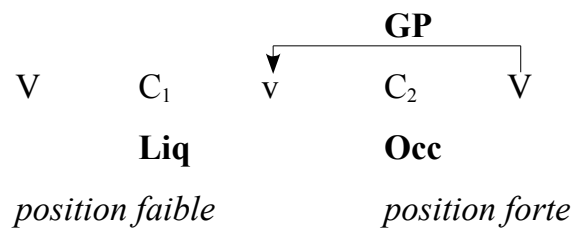

On notera que ces faits sont sensiblement les mêmes que ceux rencontrés en diachronie. Par exemple, chez (Bourciez \& Bourciez, 1967), on trouvera des formes usitées jusqu'au milieu du XVIIème s. en (10), (11) et (12) pour fr. arbre, pauvreté et mercredi :

$$
\begin{aligned}
& \text { (10) [вb] : arbre : [акььә] > a.fr. [abrə] } \\
& \text { (11) }[\text { вk] : mercredi : [merkrødi] > a.fr. [mekrødi] } \\
& \text { (12) [вt] : pauvreté : lat.[pauperte] > a.fr. [poverte] > [povьəte] }
\end{aligned}
$$

où (10) et (11) présentent le même phénomène de suppression de la liquide en ancien français. (12) présente une stratégie spécifique où la coda est déplacée en attaque. Si l'on reprend les données en (6) pour tortue, on remarque que l'une des stratégies utilisées est justement de créer une attaque branchante : [tвоty], [tьотœ]. Nous résumons ces faits dans les schémas suivants où (6a) est le cas attendu, (6b) le cas où la liquide en position faible disparaît enfin $(6 \mathrm{c})$ le cas où la liquide ne disparaît pas mais est rattachée à une attaque et forme avec la consonne qui la précède un domaine de gouvernement infra-segmental :

$$
\begin{array}{llllll}
\text { (6a) } & & \multicolumn{4}{c}{\text { GP }} \\
\mathrm{C}_{1} & \mathrm{~V}_{1} & \mathrm{C}_{2} & \mathrm{~V}_{2} & \mathrm{C}_{3} & \mathrm{~V}_{3} \\
\mathrm{t} & \text { о } & \text { в } & \varnothing & \mathrm{t} & \mathrm{y}
\end{array}
$$

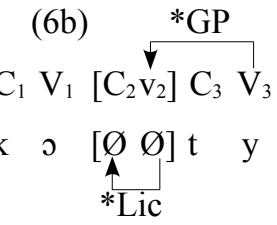

$$
\begin{aligned}
& \text { (6c) } \\
& {\left[\begin{array}{lll}
\mathrm{C}_{1} & \mathrm{~V}_{1} & \mathrm{C}_{2}
\end{array}\right] \mathrm{V}_{2} \mathrm{C}_{3} \mathrm{~V}_{3}} \\
& {\left[\begin{array}{llllll}
\mathrm{t} & \leftarrow & \mathrm{b}
\end{array}\right] \quad \mathrm{o} \quad \mathrm{t}} \\
& \text { GI Lic }
\end{aligned}
$$

Autrement dit, on retrouve, dans la paraphasie, un paramètre actif qui porte sur des conditions syllabiques et qui est similaire à ce que l'on a pu rencontrer diachroniquement en français mais qui a été synchroniquement désactivé. On retrouve ici l'hypothèse défendue par la TCSR selon laquelle les contraintes peuvent être, dans l'aphasie, fixées négativement ou positivement et que cette paramétrisation peut renvoyer à une langue, ici un état de langue, très éloignée.

La stratégie utilisée en (6c) contredit l'hypothèse, défendue par la TCSR et la Théorie de l'Optimalité, d'une simplification des structures en terme d'objets manipulés. Dans ce cas précis, la création d'un domaine de gouvernement est une stratégie réparatrice qui a le même poids que la suppression. Or si l'on en demeure à la surface, il y a certes disparition de la coda mais accompagnée d'une complexification de l'attaque : les cadres précités ne peuvent l'envisager ${ }^{7}$.

Le cas en (7) de serpent réalisé [sєьәра̃] met à jour une troisième stratégie, qui vient conforter l'analyse en terme de gouvernement, puisqu'ici apparaît la voyelle $v_{2}$ qui jusqu'alors ne pouvait s'exprimer, étant proprement gouvernée.

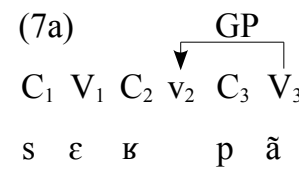

$\begin{array}{lllllll}\mathrm{C}_{1} & \mathrm{~V}_{1} & \mathrm{C}_{2} & \mathrm{~V}_{2} & \mathrm{C}_{3} & \mathrm{~V}_{3}\end{array}$

S $\quad \begin{array}{llllll}\varepsilon & \boldsymbol{\zeta} & \partial & \mathrm{p} & \tilde{\mathrm{a}}\end{array}$

Si la condition de gouvernement est rendue inopérante ( $\left.{ }^{*} \mathrm{GP}\right)$ dans le cas de l'aphasie, le réflexe est l'apparition de la voyelle par défaut : schwa. L'expression d'une unité sous-jacente devrait être confirmée 
dans d'autres cas de figure, nous nous proposons d'analyser le cas des clusters /s+occlusive/ qui, a priori, devraient exhiber le même comportement que les groupes /liquide+occlusive/.

\section{/s+occlusive/}

Les groupes / $\mathbf{b}+$ occlusive/ et /s+occlusive/ sont constitués, mise à part la catégorie de la première consonne, à l'identique. On s'attend donc à un comportement similaire. Cependant ces groupes $/ \mathrm{s}+$ occlusive/ posent problème à la phonologie ${ }^{8}$ : sont-ils hétérosyllabiques ou homosyllabiques ? L'hypothèse défendue dans le cadre de la Phonologie du Gouvernement est que ces membres sont hétérosyllabiques et séparés par un noyau vide. Nous montrons que si l'hypothèse est juste, les cas /sp/, /st/ et /sk/ ne sauraient être considérés comme équivalents.

Par exemple en $(13,14$ et 15$)$ pour les cas de moustique, casquette et aspirateur, on remarque que ces groupes peuvent être soumis à de multiples transformations :

(13) [st] : moustique [mustik] est réalisé [musik], [mosi].

(14) [sk] : casquette [kasket] est réalisé [kraket], [taset], [kakes], [taspet].

escargot [eskargo] réalisé [eskago], mais aussi [eskabõ], [kargo], [عkargo].

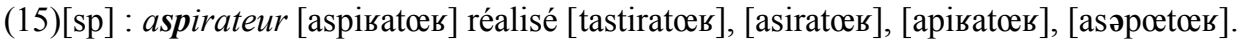

On notera, dans l'exemple (14b) suivant où escargot est réalisé [eskago] que la disparition de [в] engendre l'annulation de la condition de Gouvernement Propre comme dans le cas $(6 \mathrm{~b})$ précédent. Cette stratégie est disponible pour [s] dans les mêmes conditions (cf. [عkasgo]). On s'attend à ce que la troisième stratégie qui annule le gouvernement propre dans le cas des groupes / $\mathbf{b}+$ occlusive/ (cf.7b précédent) puisse aussi être envisagée pour le cas de v situé entre [s] et [k]. Par conséquent, ce noyau vide devrait surfacer sous forme de schwa : or, *[esəkago] n'est jamais attesté.

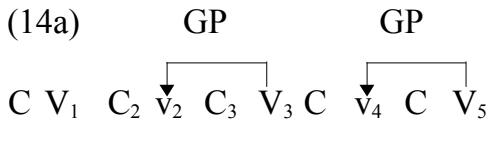

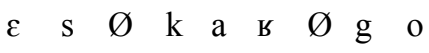

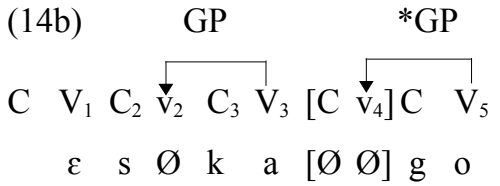

Il en va de même pour les groupes [st] qui correspondent dans ce cadre à $/ \mathrm{sVt} /$, et pour lesquels dans l'ensemble de notre corpus, il n'apparaît jamais de voyelle « épenthétique ». Seul le groupe [sp] atteste de la présence d'une position vocalique qui surface : aspirateur [аspікатœь] est réalisé [аsəрœеюь].

$$
\begin{aligned}
& \text { (15a) GP (15b) *GP }
\end{aligned}
$$

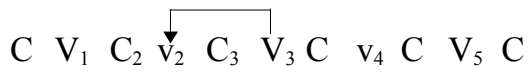

$$
\begin{aligned}
& \begin{array}{llllllllllllllll}
\mathrm{C} & \mathrm{V}_{1} & \mathrm{C}_{2} & \bar{v}_{2} & \mathrm{C}_{3} & \mathrm{~V}_{3} & \mathrm{C} & \mathrm{v}_{4} & \mathrm{C} & \mathrm{V}_{5}
\end{array}
\end{aligned}
$$

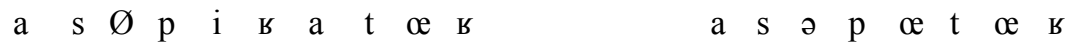

Nous postulons que ce type de cluster est sous l'effet du Principe du Contour Obligatoire (PCO), dans les cas de [st] et [sk], et qui ne peut opérer dans le cas de [sp] et qui est dû à la structure élémentaire particulière de ces groupes.

(16) [sp] / [st] / [sk] et le PCO

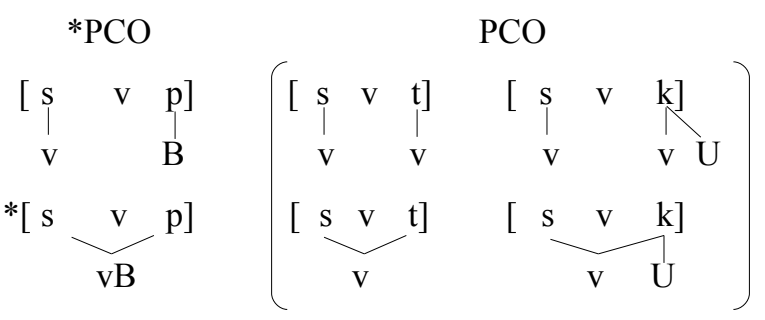


En effet dans le schéma précédent, [st] et [sk] possèdent des éléments communs (sur lesquels nous allons revenir) qui, dans cette configuration, sont soumis au PCO. La position vocalique interconsonantique n'est pas disponible : elle est bloquée par l'association des auto-segments (des éléments) partagés. Dans le cas de [sp], aucun élément n'est partagé par les deux consonnes du groupe : le PCO est inopérant et, de fait, ne bloque pas l'accès à la voyelle interconsonantique qui peut apparaître.

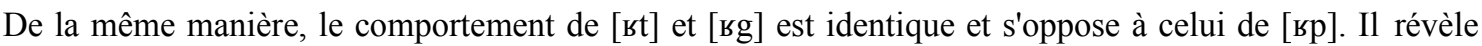
différents degrés de complexité des occlusives dont la structure interne détermine la transformation. L'analyse montre pourquoi les «substitutions» de segments répondent systématiquement à un même schéma de lénition, que ce soit dans le cas de /s+occlusive/ ou de / $\mathrm{b}+$ occlusive/.

\subsection{Marque-Complexité}

Dans cet exemple des groupes /s+occlusive/, les conditions syllabiques (position forte / position faible, gouvernement et licenciement) rejoignent les contraintes segmentales (structure élémentaire, PCO). Ces paramètres interdépendants renvoient à la question de la complexité. Cette dernière a toujours été envisagée au filtre de la Marque. Si un segment est marqué, il sera rare d'un point de vue typologique, acquis tardivement par l'enfant et perdu tôt par l'aphasique (Jakobson, 1969). Cette hypothèse de la marque-complexité, développée dans les approches linguistiques et aphasiologiques comme on l'a vu pour l'Optimalité et la TCSR, a pour corollaire la « simplification » des structures chez le patient cérébro-lésé.

Par exemple, la Distance Interphonémique (dorénavant DI (Blumstein 1973, Valdois \& Nespoulous, 1994)) élaborée pour mesurer le degré de complexité de l'erreur produite, compare les valeurs de traits distinctifs au sein de deux matrices. Si on compare les valeurs de traits de A B et C :

$$
/ \mathrm{A} /:[+\mathrm{X},+\mathrm{Y},+\mathrm{Z}] \quad / \mathrm{B} /:[+\mathrm{X},-\mathrm{Y},+\mathrm{Z}] \quad / \mathrm{C} /:[+\mathrm{X},-\mathrm{Y},-\mathrm{Z}]
$$

On remarque que la différence entre $(A, B)(B, C)$ et $(A, C)$ est respectivement de 1,1 et 2 . Autrement dit, la distance $(A, C)$ plus importante qu'entre $(A, B)$ suppose une erreur d'une plus grande complexité. Cependant, on notera que les trois segments ont la même complexité : tous possèdent le même nombre de traits. La DI ne fait donc que constater le degré d'erreur mais elle ne permet aucunement de prédire quelles erreurs sont susceptibles d'être produites dans les paraphasies phonémiques.

Les cadres qui manipulent des primitives unaires, construits sur une critique du binarisme, semblent plus à même de rendre compte de la complexité et de prédire les transformations parce que la complexité découle du nombre d'éléments en jeu. Chaque unité segmentale se compose d'un certain nombre d'éléments : ainsi chez Harris (1994), Scheer (1998) et Angoujard (1997), nous aurons les représentations suivantes pour les coronales (notées $/ \mathrm{T} /$ ), les labiales (notées $/ \mathrm{P} /$ ) et les dorsales $(/ \mathrm{K} /)$ :

\begin{tabular}{|c|c|c|c|}
\hline & $/ \mathrm{T} /$ & $/ \mathrm{P} /$ & $/ \mathrm{K} /$ \\
\hline (1994): & $\{h R P\}$ & $\{\mathrm{hUP}\}$ & $\{h v P\}$ \\
\hline Scheer (1998): & $\{h v ?\}$ & $\{\mathrm{hBP}\}$ & $\{h U v P\}^{9}$ \\
\hline Angoujard (1997) : & $\{v I P\}$ & $\{v U P\}$ & $\{\mathrm{V} P\}^{10}$ \\
\hline
\end{tabular}

On notera que les représentations proposées ne supposent pas le même nombre d'éléments pour les dorsales qui apparaîtront donc plus complexes. La prédiction est que plus un segment comporte d'éléments, plus il a de chances de se voir transformé. Nous avons montré que pour les groupes, les transformations ne sont pas aléatoires mais contraintes par les relations de gouvernement. Si le comportement de [sp] n'est pas identique à celui de [st] et [sk], alors il doit en être de même concernant les unités à l'état isolé. Autrement dit, les comportements de [p], [t] et [k] ne seront pas identiques. Nos données le confirment : nous observons que les trois classes coronale $/ \mathrm{T} /$, labiale $/ \mathrm{P} /$ et dorsale $/ \mathrm{K} /$ ne présentent effectivement pas la même phénoménologie dans l'aphasie. Cependant, une régularité se dégage : 
(17) Lénition des obstruantes

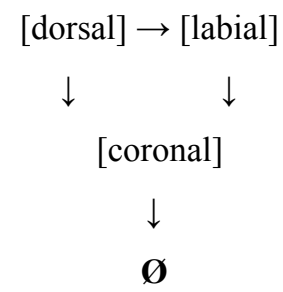

- $\quad$ si /K/ se transforme le plus souvent en $/ \mathrm{P} / \mathrm{et} / \mathrm{ou} / \mathrm{T} /$,

- $\quad / \mathrm{P} /$ se transforme en $/ \mathrm{T} /$,

- $\quad / T /$, quant à lui, ne peut que disparaître.

En résumé, les dorsales sont capables de "tout", tandis que les labiales ne peuvent se transformer qu'en coronale, et /T/ est le résultat ultime avant la disparition. Si l'hypothèse est juste, la structure interne de ces segments doit rendre compte du déséquilibre où $/ \mathrm{K} /$ est une unité plus complexe que $/ \mathrm{P} /$ et $/ \mathrm{T} /$ et où $/ \mathrm{P} /$ reste plus complexe que $/ \mathrm{T} /$. L'hypothèse de la $\mathrm{DI}$, dans un cadre binariste, n'est pas en mesure de rendre compte de ce comportement contrairement au cadre unariste. Nous considérerons avec Scheer (1998) que les coronales doivent être envisagées comme ayant la structure interne la moins complexe et les dorsales, la structure la plus complexe. A présent, il faut continuer à explorer cette voie où il est nécessaire de rendre compte de la position intermédiaire des labiales qui, dans les cadres phonologiques actuels, comportent autant de primitives que les coronales. Autrement dit, il ne nous est pas possible, à ce stade, de différencier les comportements de ces deux classes sur la base de leur structure interne. Nous ne doutons pas que les recherches futures permettront d'élucider cette asymétrie.

\section{Discussion}

Nous avons montré, dans un cadre aphasiologique, que la TCSR ne saurait être confondue avec la Théorie de l'Optimalité même si ces deux cadres exploitent la notion de contrainte. Nous avons expliqué que l'approche optimaliste, outre le fait qu'elle use de contraintes insuffisamment restrictives pour traiter de l'aphasie, repose sur l'hypothèse d'une contiguïté séquentielle et achoppe à décrire la complexité structurelle et segmentale qui constitue le coeur du trouble aphasique. Par ailleurs, ces deux cadres développent parallèlement une mesure de la complexité qui, nous l'avons souligné, repose exclusivement sur un décompte des objets en jeu. De fait, ils sont inadaptés pour répondre aux transformations fréquentes dans les aphasies : pourquoi certaines réalisations aphasiques présentent a priori un accroissement en complexité ? Qu'est-ce qui est à l'origine du déséquilibre entre attaque et coda branchantes ? Comment rendre compte de la structure interne des segments qui soit compatible avec leur comportement syllabique?

L'approche qualitative que nous avons développée dans le cadre de la Phonologie du Gouvernement apporte une solution à la question des clusters consonantiques et de leur rôle dans la définition de la complexité segmentale et syllabique. Ce changement de paradigme amène à reconsidérer cette notion de complexité en terme d'opérations conditionnées par le gouvernement, le licenciement et la structure interne des segments. Ce modèle, en apportant de nouvelles solutions, ouvre de nouvelles perspectives d'analyse des comportements aphasiques.

Les travaux en acquisition développent également des hypothèses sur le comportement des clusters (Demuth \& Kehoe, 2006), (Kirk, 2008), (Kehoe \& Hilaire Debove, 2004) ou encore (Goad, 2011) qui conduisent à des résultats similaires à ce que l'on peut trouver en aphasiologie. Cependant, ces travaux en acquisition sont exposés aux mêmes questions et problèmes non-résolus. Même si les données récoltées pour l'instant semblent aller dans le sens du postulat jakobsonien et démontrer le parallèle entre acquisition et régression du langage, il s'avère nécessaire à présent de sélectionner pour l'analyse des objets comparables dans les deux champs. Un protocole pilote commun aux enfants et aux aphasiques, 
concernant les clusters / $\mathbf{b}+$ occlusive/ et /s+occlusive/, est actuellement testé. Dans la continuité du présent travail, ce protocole permettra d'envisager la complexité tant dans le domaine de l'acquisition que dans celui de l'aphasiologie. Dans cette optique, l'aphasie constitue au même titre que le langage nonpathologique et l'acquisition un terrain d'expérimentation propice pour interroger la validité des modèles phonologiques.

\section{Références bibliographiques}

Angoujard, J-P. (1997). Théorie de la Syllabe. Rythme et Qualité. Paris : CNRS Editions.

Béland, R. \& Nikiema E. (1993). Historique de la notion de "Contrainte" en phonologie générative. Langues et Linguistique, vol. 19, 45-70.

Béland, R. \& Paradis, C. (1997). Principled syllabic dissolution in a primary progressive aphasia case. Aphasiology, vol.12, 1171-1196.

Béland, R. Paradis, C. McClish, I \& Macoir, J. (2001) Traitement syllabique et prédictions dans un cas d'aphasie progressive primaire. Langues et Linguistique, vol. 27, 57-94.

Blumstein, S. (1973). A phonological investigation of aphasic speech. Janua Linguarum Series Minor, vol.153, The Hague : Mouton.

Bourciez, E. \& Bourciez, J. (1967). Phonétique française, étude historique. Paris : Klincksieck.

Brandão De Carvalho, J. \& Wauquier, S. (2007). Approches inductives et théorie phonologique : vrais et faux problèmes. Recherches linguistiques de Vincennes, vol. 36, 37-68.

Demuth, K. \& Kehoe, M. (2006). The acquisition of Word-final Clusters in French. Catalan Journal of Linguistics , vol. $5,59-81$

Den Ouden, D-B. (2002). Phonology in Aphasia, syllables and segments in level-specific deficits. Thèse de Doctorat, Grodil 39 : Universiteit Groningen.

Goad, H. (2011) sC Clusters are Coda-initial. In van Oostendorp M., Ewen C., Hume E. \& Rice K. (eds.) The Blackwell companion to phonology. Oxford : Blackwell, 898-923.

Goldsmith, J. (1990). Autosegmental and metrical phonology. Oxford : Blackwell.

Harris, J. (1994). English sound structure. Oxford : Blackwell.

Jakobson, R. (1969). Langage enfantin et aphasie. Paris : Editions de Minuit.

Kaye, J., \& Lowenstamm J. (1981). Syllable structure and Markedness Theory. in Belletti A. \& al. (eds), Theory of Markedness in Generative Grammar, Pisa : Scuola Normale, 287-315.

Kaye, J., Lowenstamm, J. \& Vergnaud, J.R. (1985). The internal structure of Phonological Elements : Theory of Charm and Government in C. Ewen et J. Anderson (eds) : Phonology Yearbook, 2. Cambridge : Cambridge University Press, 305-328.

Kaye, J., Lowenstamm, J. \& Vergnaud, J.R. (1990). Constituent structure and government in phonology. in Phonology yearbook 7. Cambridge : Cambridge University Press, 193-231.

Kaye, J. (1998). Do you believe in magic ? The story of s+C sequences, SOAS : WPLP, 293-313.

Kehoe, M., Hilaire Debove, G., Demuth, K. \& Lleo, C. (2008). The structure of Branching Onsets and Rising Diphtongs: Evidence from the Acquisition of French and Spanish. Language Acquisition. A journal of Developmental Linguistics, vol. 15.1, 5-57.

Kirk, C. (2008). Substitution Errors in the Production of Word-initial and Word-final Consonant Clusters. Journal of Speech Language and Hearing Research, vol. 51, 35-48.

Laks, B. (2005). Approches cognitives de la phonologie in V. Rey, N. Nguyen (eds.) : Nouvelles approches en phonétique et en phonologie. Paris : Hermès, 291-319. 
Lowenstamm, J. (1996). CV as the only syllable type in Durand J. \& Laks B. Current trends in phonology models and methods. Paris X : CNRS, 419-442.

Moreau, N. (1993). Apport de la théorie « des contraintes et des stratégies de réparation » à l'étude des paraphasies phonémiques dans l'aphasie. Thèse de doctorat : Université de Toulouse-le-Mirail.

Nespoulous, J-L. (2006). Le langage et les processus cérébraux : apport de la linguistique et de la psycholinguistique à l'aphasiologie et à la neuropsycholinguistique cognitive du XXème siècle, in S. Auroux \& al. (Eds.) History of Language Sciences. Berlin / New- York : Mouton de Gruyter, 2671- 2682.

Prince, T. (2011). Aphasie : processus de détérioration phonologique : déconstruction des clusters consonantiques et syllabicité. Mémoire de Master : Université de Nantes.

Scheer, T. (1998). A theory of consonantal interaction. Langues et grammaire II \& III : Phonologie, 141-172.

Scheer, T. (2000a). De la localité, de la morphologie et de la phonologie en phonologie. Habilitation à diriger des recherches : Université de Nice, 1-366.

Scheer, T. (2004). A lateral Theory of Phonology : What is CVCV and what it should be?. Berlin : Mouton de Gruyter.

Scheer, T. \& Brun-Trigaug G. (2010). Lenition : branching onsets in French and in ALF dialects. In Karlík, P. (ed)

Development of Language through the Lens of Formal Linguistics, Munich : Lincom, 15-28.

Scheer, T. \& Zikova, M. (2010). The Coda Mirror v2. Tours : Réseau Français de Phonologie.

Selkirk, E. (1984). Phonology and syntax : the relation between sound and structure. Cambridge : MIT press.

Valdois, S. (1987). Les erreurs d'addition et d'omission dans l'aphasie : rôle du gouvernement phonologique. Thèse de doctorat : Université de Montréal.

Valdois, S. Nespoulous, J.-L. (1994). Perturbations du traitement phonétique et phonologique du langage in Seron X. Jeannerod M. Neuropsychologie Humaine. Bruxelles : Mardaga, 360-374.

Valdois, S. (2006). Les transformations segmentales d'origine aphasique in Eustache, F. \& Lechevalier, B. : Langage et aphasie. Bruxelles : De Boeck, 107-125.

Wilshire, C. \& Nespoulous, J-L. (2003). Syllables as Units in Speech Production : Data from Aphasia in Brain and Language. Elsevier Science Academic Press, 424-447. 
${ }^{1}$. Béland, Paradis, McClish \& Macoir (1997: 6-7).

${ }^{2}$.Le niveau le plus bas dont fait mention le principe de minimalité est établi en fonction de la hiérarchie des niveaux phonologiques (dorénavant HNP) : (i) métrique $>$ syllabique $>$ squelette $>$ noeud de base $>$ articulateur $>$ trait terminal

${ }^{3}$. N. Moreau $(1993: 220)$

${ }^{4}$. Den Ouden (2002) montre à travers la Gradual Learning Algorithm que les contraintes opérantes ont un poids différent selon la nature de l'aphasie : fluentes ou non-fluentes. Ces contraintes engendrent de fait des données et des résultats différents selon la hiérarchie dans laquelle elles sont placées.

5 . Prince (2011).

${ }^{6}$. Pour ces cas, nous renvoyons à Prince (2011 : 130 et suiv.) qui propose un traitement en terme d'harmonie (diffusion d'éléments).

7. Ce passage de coda à attaque branchante contredit la Marque où « The presence of an onset of a given language degree of markedness implies the presence of a rime of an equal degree of markedness in that language. $(\ldots)(\mathrm{m}, \mathrm{n})$ where $\mathrm{m}$ is the maximum markedness value for the onsets and $\mathrm{n}$ is the maximum markedness value for the rime, moreover $\mathrm{m} \leq \mathrm{n}$. » Kaye \& Lowenstamm (1981 : 294-295). Cependant, nous ne postulons pas que toutes les codas répondent à ce même principe, nous postulons que la stratégie adoptée par les aphasiques dans le cas des groupes / $\mathbf{b}+\mathrm{occ} /$ est de déplacer la liquide de sa position coda en une position d'attaque branchante. Ceci démontre le statut particulier de la liquide / $/$ / sans remettre en question les analyses traditionnelles sur la marque. Certes la position attaque branchante est définie comme moins complexe que la coda, toutefois, lorsque l'aphasique est confronté à/b/ en coda au sein d'un cluster, celui-ci lui apparaît comme plus complexe.

${ }^{8}$. Voir notamment les traitements pionniers de Selkirk (1984) ou Kaye (1998).

${ }^{9}$. Où $<\mathrm{B}>$ renvoie à la labialité, $<\mathrm{U}>$ à la vélarité, $<\mathrm{v}>$ à la position de repos, $<\mathrm{h}>$ au bruit et $<\mathrm{P}>$ à l'occlusion.

${ }^{10}$. Où $<$ I $>$ représente la coronalité, $<\mathrm{U}>$ la labialité et $<\mathrm{v}>$ la dorsalité. 